\section{Ethical responsibility and treatment errors}

J. Blood

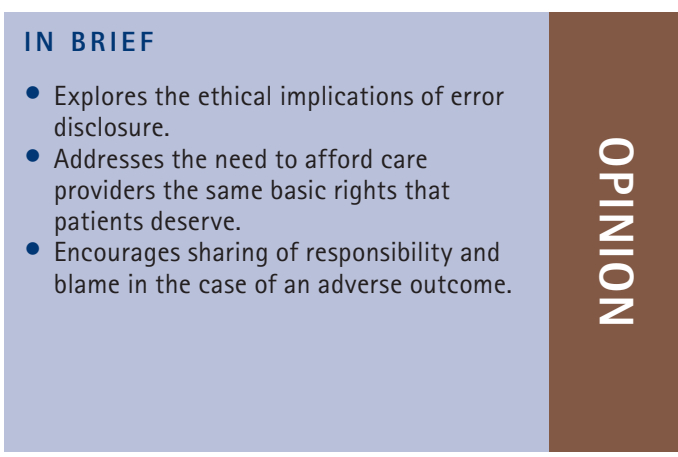

\begin{abstract}
Error disclosure is an important part of a healthcare provider's job. In a system such as healthcare, it is inevitable that mistakes will be made and that patients will suffer unnecessarily. Knowing this raises many questions. How does one determine when and how much to disclose? Is there such a thing as too much honesty? With all of the conflicting ideas about when, where, how and what to disclose, how can a provider decide for him or herself how to handle each situation? And should the provider be held responsible for the error or should the system or the patient accept some of the blame in many cases? This article will explore the factors influencing how errors are disclosed and will discuss the idea of responsibility in medical care and error disclosure.
\end{abstract}

Between every speciality and within each speciality, there is a vast difference in opinions as to how error disclosure should be approached. Loren et al. pointed out that physicians are less likely to disclose errors but are more likely to apologise during a disclosure, whereas risk managers are more supportive of disclosing errors but less likely to encourage the physician to provide a full apology to the patient. ${ }^{1}$ Doctors experience 'enormous pressures' from patient advocates and ethicists to give a full disclosure while dealing with demands from liability insurers and risk managers who expect the doctor to practise restraint in disclosure. ${ }^{2}$

There is even more disagreement between providers and other parties when it comes to errors that do not result in any obvious harm. Ethically, it is often agreed that the care provider is obligated to respect patient autonomy and reveal mistakes if the mistake 'significantly affects the patient's health and well-being, ${ }^{3}$ But how does one determine the significance of an error? Physicians are often less inclined to disclose errors that have not resulted in harm and it has been noted that their decision to disclose may be based on their perception of whether the event is actually an error and how much harm the patient has experienced. ${ }^{4}$ However, as Chamberlain et al. pointed out, physicians

'DDS, Independent Contractor, 328 Waterloo Street, Lawrenceburg, USA

Correspondence to: Dr Jamie Blood

Email:jherra@llu.edu

\section{Refereed Paper}

Accepted 25 October 2013

DOI: $10.1038 /$ sj.bdj.2014.4

${ }^{\circledR}$ British Dental Journal 2014; 216: 59-60 are often 'poor at predicting future medical outcomes' due to the vast number of influential factors in each case. ${ }^{4}$

Some authors believe that the fear of legal action or the fact that the patient may never become aware of the mistake does not justify keeping information from the patient and that by not giving a full disclosure, the provider may be implicating their profession as one that should not be trusted. ${ }^{3}$ And yet, with each difficult disclosure situation, the consequences of the doctor's decision are often theirs and theirs alone. As Ghalandarpoorattar et al. showed, assigning blame to individuals is often the "dominant culture in medical error management. ${ }^{5}$ This application of blame occurs despite the fact that the majority of adverse events arise due to multiple factors that no individual should be blamed for. ${ }^{6}$ If the provider chooses to give a full disclosure, they may lose the support of the risk managers and insurers and put themselves in a position to have a lawsuit brought against him or her. On the other hand, if they choose to withhold some information and the patient finds out, they risk a lawsuit for not giving full disclosure as well as the wrath of advocates and ethicists. They are stuck in the middle and punished for making the 'wrong' decision, even though nobody can agree on the 'right' decision. The provider's decision is further complicated by the blame culture that we see in today's 'litigation society' which makes it even more difficult to distinguish between negligence and an unavoidable adverse outcome. ${ }^{6}$

Recent changes in error management do appear to be exerting a positive effect on the dilemma of error disclosure. In a recent study, it was found that the number of interns willing to fully disclose their mistakes has increased significantly over the last decade (whether or not the patient experienced any adverse effects), while their fear of litigation after disclosure has steadily decreased. ${ }^{7}$ It was also noted that the number of interns who felt that mistakes were preventable if the doctor knew enough had decreased and the interns felt more strongly that competent doctors should not have to keep emotions and uncertainties to themselves and should not have to leave medicine if they made an error that resulted in harm to the patient or death. ${ }^{7}$ Varjavand et al. suggested that significantly more prior training regarding medical mistakes could be the reason for these changes in attitude. ${ }^{7}$

While a lot of focus has been placed on the effect that an error disclosure may have on the patient, the effect on the care provider is often overlooked. The patient has, of course, been subjected to harm and their feelings are undeniably important and must be considered. Communication with the patient can either create or prevent increased trauma. ${ }^{2}$ At the same time, shouldn't we also give more consideration to the feelings of the care provider? We are all human and we all make mistakes. Can we expect providers to give a full disclosure to their patient when we don't teach them how to disclose or how to deal with the emotions of that disclosure? Is it fair to expect a complete confession when providers feel like they have no one to support them and they are alone in their decision and alone in suffering the consequences?

Some believe that the interests of the wronged patient should completely trump 
those of the care provider, and that the idea that admitting mistakes can be painfully difficult for professionals is a 'trite' observation. ${ }^{7}$ Hannawa pointed out that an error disclosure is often not just difficult, but an 'overwhelming challenge' for providers. ${ }^{2}$ Providers are made to feel throughout their training that they are to be held to a higher standard and that mistakes are unacceptable. It is almost inevitable then that they feel like failures when an error does occur and the fear of failing can impact how much they are willing to extend themselves professionally. ${ }^{9}$ This feeling of failure after an error occurs, combined with the strong emotions in a disclosure conversation, as well as a fear of legal repercussions, all can have a devastating impact on the care provider. And yet, providers are expected to conduct these disclosures in just the right way so that the patient feels better. As Hannawa showed through his research, error disclosures that do occur often fail to meet patient expectations and there is a large gap between what the patients want to hear and what the physicians tell them. ${ }^{2}$ Despite the fact that every patient is different and would prefer to be communicated with in a different way, physicians are often expected to figure out what their specific patient wants to hear and are held responsible when they do not meet that patient's expectations.

A study conducted in China revealed that many respondents believed that the physician who made a medical error should be punished by possibly having his or her licence revoked. ${ }^{10}$ Life-changing mistakes can be devastating. However, it seems that many patients do not understand the commitment and dedication that is required to become a care provider. A physician who has devoted a decade of their life to learning their profession should not lose everything because of an unintentional error. How many patients would be comfortable with the idea of losing their jobs every time they made a mistake? How many people would agree to give up their driving licence for the rest of their lives if they ever ignored the speed limit or distractedly ran a traffic light and caused an accident? Physician errors often have very devastating effects because physicians make much more difficult decisions on a day-to-day basis. While they should be held to a higher standard and should be expected to make a greater effort to prevent errors, should they be held accountable to a much greater degree than any other human capable of making an unintentional error? This question was partially addressed in the study conducted by Kohn et al. that recognised that harm was not done 'deliberately, negligently or through incompetence, but through normally competent clinicians working in inadequate systems. ${ }^{11}$

In addition to seemingly unrealistic expectations and arguably devastating ideas of fair punishment, one must also consider the patient's role in providing honest, complete information to their care provider. Shekar et al. explored the 'serious imbalances in which patients' rights and corresponding ethical duties of professionals predominate over the responsibilities of patients themselves. ${ }^{6}$ If a provider is expected to fully disclose any errors, shouldn't the patient be expected to fully disclose any pertinent medical history that may have an effect on their treatment and treatment outcomes? The patient should assume that any questions asked by their provider are relevant and demand a complete answer. Yet many patients intentionally do not provide necessary information to their doctors, especially in regards to sexually transmitted diseases and recreational drug use. This lack of trust in the provider is often excused by the claim that the patient was afraid of being discriminated against. While this is a valid concern, it does not necessarily make it acceptable to partially inform the provider. How many adverse events occur because the patient did not disclose important information?

In an interview with an anaesthesiology resident, the resident related how nearly every patient that he saw at a nearby county hospital denied use of any methamphetamines. However, according to the resident, when he informed the patients that the medications he would use to anaesthetise them during surgery could cause death if mixed with amphetamines, the majority of patients would undergo a change of heart and admit that they had used these drugs mere hours before the conversation. ${ }^{12}$ As dental students, we are taught that, despite what the patient says, if we see signs of a disease we are to suspect that the disease is present. For instance, if we see an oral lesion that is pathognomonic for AIDS but the patient claims that they have made no lifestyle choices that would put them at risk for the disease or that they have been tested recently and are not HIV positive, we are still accountable for modifying the treatment plan to account for AIDS (for instance, the patient is likely at increased risk for infection due to immunosuppression so antibiotic recommendations may be altered) and for encouraging the patient to return to their doctor for further follow up and care.
If the patient does not give a complete history, there may be adverse outcomes that could have been prevented. An example in dentistry would be the use of bisphosphonates. If the patient has been on these medications, they are much more likely to experience osteonecrosis of the jaw after extractions. If the dentist knows that the patient has used these medications, he or she may opt to perform an endodontic procedure rather than an extraction to avoid this adverse effect. If the dentist is unaware, the extraction would be performed and the patient may blame the dentist when osteonecrosis occurs. While provider errors obviously cannot be prevented by the patient, perhaps it is time that some patients begin to take more responsibility for their care and for the outcomes they experience.

Medical errors that could have been avoided are devastating and every effort should be made to prevent these errors from occurring. Honesty in error disclosure is of vital importance in most situations so that the patient can make fully informed decisions. Healthcare providers should be trained to deal with these situations and patients should be educated in order to improve their ability to form realistic expectations about their medical treatment and their healthcare providers. Patients should be strongly encouraged to take responsibility for their own health and care. Finally, the very rights that the patient demands (right to full disclosure, right to have their feelings respected, right to seek restitution, right to be human and make mistakes) should be afforded to their care providers as well.

1. Loren D J, Garbutt J, Dunagan W C et al. Risk managers, physicians, and disclosure of harmful medical errors. Jt Comm J Qual Patient Saf2010; 36: 101-108.

2. Hannawa A. Communication with patients about medical incidents. Ther Umsch 2012; 69: 363-366.

3. Chiodo G T, Tolle, S W, Crichlow C. Disclosure of mistakes. Gen Dent 1999; 47: 24-28.

4. Chamberlain C J, Koniaris L G, Wu A W, Pawlik T M. Disclosure of 'nonharmful' medical errors and other events. Arch Surg 2012; 147: 282-286.

5. Ghalandarpoorattar S M, Kaviani A, Asghari F. Medical error disclosure: the gap between attitude and practice. Postgrad Med J 2012; 88: 130-133.

1. Shekar V, Singh M, Shekar K, Brennan P. Clinical negligence and duty of candour. Br J Oral Maxillofac Surg 2011; 49: 593-596.

7. Varjavand, N, Bachegowda L.S, Gracely, E, Novack $\mathrm{DH}$. Changes in intern attitudes toward medical error and disclosure. Med Educ 2012; 46: 668-677.

8. Sokol D K. The hardest thing: admitting error. BMJ 2012; 344: e3085.

9. Marshall M L. Mistakes. The nexus to growth and learning. CDS Rev 1998; 91: 22-24.

10. Gu X, Itoh K. Patient views and attitudes to physician's actions after medical errors in China. J Patient Saf 2012; 8: 153-160.

11. Kohn L, Corrigan, J M, Donaldson M S (eds). To err is human: building a safer health system. Washington DC: National Academy Press, 1999.

12. Herra D. Personal communication. 16 July 2012. 